\title{
Einige kurze Bemerkungen über die Ausscheidung und Thermometamorphose der Zechsteinsalze nach der Auffassung von Rózsa.
}

\author{
Von ERNST JÄNECKE.
}

In einem kürzlich in dieser Zeitschrift erschienenen Aufsatze ${ }^{1}$ wandte sich Rózsa gegen die Theorie, nach welcher bei der Entstehung der Kalilager sich Kainit und Bischofit primär ausgeschieden haben. Es ist dies der Gegenstand, den ich auch in meinem Buche über die Entstehung der Kalilager kürzlich behandelt habe. ${ }^{2}$ In Übereinstimmung mit anderen ist daselbst dargelegt, da $B$ die Entstehung unserer Kalilager mit Hilfe weniger geologischer und physikalisch-chemischer Annahmen in seinen Grundlagen einwandfrei zu erklären ist. Diese allgemein anerkannten Annahmen sind folgende: die Kalilager sind aus Meerwasser von der Zusammensetzung des jetzigen ${ }^{3}$ durch Verdunsten entstanden, sie sind von anderen Schichten überlagert, haben dadurch in den folgenden geologischen Zeiträumen ein Einsinken in die Erde erfahren und an den tektonischen Veränderungen der Erde teilgenommen und sind seblieBlich beim Abtragen der bedeckenden Schichten der Erdoberfläche wieder näher gekommen. Die sich hieraus mit Notwendigkeit ergebende Erwärmung und Wiederabkühlung bedingt Veränderungen physikalischchemischer Art, die eingehend besprochen wurden. An einigen Profilen wurde ganz kurz gezeigt, welche ausgezeichnete Übereinstimmung dieser Erklärung mit den geologischen Befunden (vgl. z. B. S. 93) besteht.

Rózsa wendet sich nun gegen die aus diesen Betrachtungen sich notwendigerweise ergebende Annahme primärer Kainit- und Bischofitlager und gegen meine angebliche Behauptung, da $B$ erst oberhalb $117^{\circ}$ in einer Tiefe von $3500-5000 \mathrm{~m}$ eine Umwandlung der primären Lager stattgefunden habe. Rózsa scheint mein Buch nur flüchtig durchgesehen zu haben, denn auf $\mathrm{S} .79$ habe ich ausdrücklich in gesperrtem Druck darauf hingewiesen, daß schon

12 . anorg. $u$. allg. Chem. 97 (1916), 41-55.

Verl. von Friedr. Vieweg \& Sohn, Braunschweig 1915.

3 Vgl. Watther, Geschichte der Erde und des Lebens. 1908, S. 69 u. 372.

Z. anorg. u. allg. Chem. Bd. 99. 
bei Temperaturen unter $48^{\circ}$, also einer Tiefe von weniger als $1000 \mathrm{~m}$ eine Umbildung aller primär ausgeschiedenen Salzschichten stattgefunden haben muB. ${ }^{1}$ Die Annahme der Überlagerung des Zechsteins von nur einem Kilometer anderer Schichten dürfte wohl von wissenschaftlicher Seite nirgends Widerspruch finden. Sind doch bekanntlich die Salze an dem Ende des paläozoisehen Zeitalters zur Ausscheidung gelangt und wird vielfach die Dicke jeder einzelnen der folgenden fünf Schichten: Trias, Jura, Kreide, Tertiär, Diluvium auf Kilometer geschätzt, so daB die Überlagerung aus einer Sehicht bereits mehr als $1 \mathrm{~km}$ ergeben könnte (vgl. z. B. KaYser, Geologie, 2 Bd.).

Rózsa will nun Kieserit und Carnallit als primäre Lagernngen aufgefaßt wissen und glaubt dieses durch ,chlormagnesiumreiche Zuflüsse und Endlaugen während des Eintrockungsprozesses" erklären zu können. Diese Chlormagnesiumlaugen müssen selbst schon aber auch irgendwie entstanden sein. AuBerdem muß das Chlormagnesium, nachdem es zur Bildung des Carnallits (und nicht Kainits) Veranlassung gegeben hat, wieder verschwinden, da es in der Lauge bleibt und nach Rózsa kein Bischofit entstehen soll. Eine Erklärung für dieses Woher und Wohin dürfte sehr schwierig sein. Macht man sich einmal auf Grund der bekannten Löslichkeitsverhältnisse die Mengen klar, die dazu gehören, um die primäre Bildung von Kainit zu umgehen, so kommt man zu Werten, die in der Größenordnung ähnlich den vorhandenen Salzmassen sind. Es müßten also andore Meere von Chlormagnesiumlauge dem verdunstenden Meerwasser zugeführt werden, um die primäre Bildung von Kainit zu umgehen und nur Carnallit als primäre Ausscheidung von kaliumhaltigen Salzen zu erhalten. Woher stammen diese Mengen?

In bezug auf die Verneinung von ursprünglichen primären Bischofitlagern, stimmt Rózsa mit der früher viel vertretenden Ansicht überein, wonach die Salze auch jetzt in der gleichen Form wiedergefunden würden, in der sie sich ursprünglich ausgeschieden hatten. Sind doch die Tatsachen, die eine Umsetzung der Salze im Erdinnern fordern, erst in neuerer Zeit gebührend ins rechte Licht gesetzt worden. ${ }^{2}$ Man hatte früher die Ansicht, da das notwendigerweise beim Austrocknen der Salzseen schließlich auftretende Bischofitlager nicht gefunden wurde, daß die Lauge, als sie soweit verdunstet war, weggelaufen sei. An einzelnen Stellen mußte man

${ }^{1}$ Dieses hat auch Prof. Erdmand in einer Besprechung meines Buches übersehen. Deutsche Literaturzeitung 1916, S. 2094.

2 Z. B. von Rinne, Arrhenius, Lachmann u. a. 
diese Annahme sogar zweimal machen. Dieses Weglaufen der ,Endlauge" konnte natürlich, da kein Magnesiumchlorid in den dazu nötigen Mengen in späteren Schichten gefunden wurde, nur in das Weltmeer geschehen sein. Es mußte also, gerade nachdem die Verdunstung bis zu diesem Punkte gelangt war, eine Hebung der Salzmassen oder eine Senkung eines benachbarten Meeres (welches?) erfolgt sein. Außerdem durfte diese Hebung oder Senkung auch nicht zu starks sein, da ja sonst die Salzlager wieder gelöst wären. Diese Annahme, die nur einer Hypothese zuliebe gemacht ist, erscheint gezwungen. Sie entspricht auch nicht den geologischen Tatsachen. ${ }^{1}$

Die Notwendigkeit der Annahme von ursprünglichen primären Bischofitschichten ist schon früher betont ${ }^{2}$, sie ergibt sich besonders klar bei der Betrachtung des seitlichen Verlaufes der Verdunstung des Meerwassers. Nimmt man einmal willkürlich an, die Zeit von dem Beginn der Verdunstung des Zechsteinmeeres bis zu seiner Trockenlegung habe 100000 Jahre gedauert ${ }^{3}$, so kann man aus der verdunstenden Wassermenge schätzungsweise berechnen ${ }^{4}$, daß die Ausscheidung der Kalisalze hiervon nur etwa 500-1000 Jahre in Anspruch nahm, wovon wiederum etwa die Hälfte zur Ausscheidung des Bischofitlagers verbraucht wurde. In dieser geologisch sehr kurzen Periode sollte nun gexade eine Unterbrechung stattgefunden haben? Es kann geologisch als sicher gelten, daß beim allmählichen vollständigen Austrocknen des Zechsteinmeeres in dem damaligen Wüstenklima durch immer weitere Schrumpfung des Laugenbeckens die trockenen Stellen immer größer wurden, und so der Wüstenwind die austrocknenden Salze mit feinem Ton überziehen konnte. Findet man doch Ton nicht nur in dem eigentlichen Salzton, sondern auch in den Kalilagern ${ }^{5}$. Um ein primärcs Bischofidlager kommt man also auf keinen Fall herum.

In Wirklichkeit ist keiner der von Rózsa angegebenen geologischen Befunde und chemischen Analysen in Widerspruch mit den ausführlichen Darlegungen in meinem Buche. Dagegen sind manche der Bemerkungen, besonders die sich auf thysikalischchemische Umsetzung beziehen, nur halb richtig, teilweise durchaus falsch. Die chemische Gleichung z. B., die Rózsa auf S. 50 seiner Arbeit gibt:

$$
\begin{array}{r}
2 \mathrm{KCl} . \mathrm{MgCl}_{2} \cdot 6 \mathrm{H}_{2} \mathrm{O}+3 \mathrm{MgSO}_{4} \cdot \mathrm{H}_{2} \mathrm{O}+\text { Lauge } \\
\rightarrow \mathrm{K}_{2} \mathrm{Mg}_{2}\left(\mathrm{SO}_{4}\right)_{3}+3 \mathrm{MgCl}_{2}+\text { Lauge }
\end{array}
$$

${ }^{1}$ Vgl. WALTHER, 1.c. S.373. ${ }^{2}$ Z. B. von BoEKe. ${ }^{3}$ Vgl. WALTHER, l.c. S.164. 4 JÄNECKE, 1. c. S. 65 . ${ }^{5}$ Vgl. WALTHER, 1. c. S. 372 . 
ist kaum verständlich. Rein als Gleichung genommen sagt sie, daß aus einem Gemisch zweier fester Salze mit Lange zwei andere entstehen, indem die Lauge sich verdünnt. Eines der Salze ist wasserfreies Magnesiumchlorid! Es ist Rózsa offenbar nicht klar, daß die Zusammensetzung einer Lauge, die an einer Umsetzung teilnimmt, die größte Bedeutung hat und daB Gleichungen der von ihm angegebenen Art unverständlich sind. ${ }^{1}$ Außerdem ist bei solchen Umsetzungen die Temperatur und das Mengenverhältnis der festen Salze wichtig und hat man durchaus nicht nötig, künstlich Umsetzungsgleichungen zu konstruieren. Die wirklichen Umsetzungen sind durch die vielen Untersuchungen der letzten Zeit recht gut bekannt.

Eine Behauptung, die den physikalisch-chemischen Untersuchungen (VAN'T Hoffs) widerspricht, steht auf S. 48. Dort behauptet Rózsa, daß aus Kainit theoretisch bei der Umwandlung genau eine äquimolekulare Menge Carnallit und Kieserit entstehen müsse. Er meint, dieses sei auch meine Ansicht. Dabei habe ich in meinem Buche auf vier Seiten ${ }^{2}$ diesen Vorgang, der von VAN'T HoFr sehr genau untersucht wurde, eingehend behandelt. Aus Kainit entsteht Langbeinit neben Sylvin und Kieserit, und letztere beiden nicht in äquimolekularem Verhältnis.

\section{Zusammenfassung.}

Die von Rózsa bestrittenen primären Kainit- und Bischofitausscheidungen bei der Entstehung unserer Kalilager sind notwendige Folgen der allgemein anerkannten Theorie der Entstehung aus Meerwasser. Die von Rózsa ad hoc gemachte Annahme von Zuflüssen von Magnesiumchloridlaugen führt zu der nicht beantworteten Frage nach dem Entstehen und Bleiben dieser Salze. Es sind daher diese und andere Angriffe Rózsas gegen die Darlegungen in meinem Buche iuber die Entstehung der Kalilager unberechtigt. Einzelne Angriffe Rózsas richten sich gegen Behauptungen, die überhaupt nicht gemacht wurden. In einzelnen Fällen wurde das gerade Gegenteil ausführlich auseinandergesetzt. Die physikalisch-chemischen Erörterungen Rózsas widersprechen mehrfach den Gesetzen der Umsetzung fester Salze in Gegenwart von Laugen.

1 Es finden sich in seinen letzten Arbeiten noch mehr solche. Vgl. z. B, Beibl. d. Ann, 1916. S. 304.

S. $49-62$.

Hannover, Kgl. Technische Hochschule, im Oktober 1916.

Bei der Redaktion eingegangen am 3. Oktober 1916. 